\title{
Controlling the drying-induced peeling of colloidal films
}

Abdulkadir Osman (1), Lucas Goehring (2), Hugh Stitt (3), Nima Shokri* (4)

(1) Department of Chemical Engineering and Analytical Science, The University of Manchester, Manchester M13 9PL, United Kingdom

(2) School of Science and Technology, Nottingham Trent University, Clifton Lane, Nottingham NG11 8NS, UK

(3) Johnson Matthey Technology Centre, Billingham TS23 1LB, UK

(4) School of Civil Engineering, Hamburg University of Technology, Hamburg, Germany

*Corresponding author: nima.shokri@tuhh.de

\begin{abstract}
In this work, we investigated the effect of the suspension properties on the drying dynamics and the resulting film peeling instability. To do so, a comprehensive series of experiments were conducted using drops of aqueous mixtures of colloidal silica dispersions and polyethylene oxide (PEO) additives. Time-lapse digital microscope images of the evaporating droplets shows that film peeling can be discouraged and eventually eliminated with an increase in PEO concentration and molecular weight. This is due to the additives modifying the suspension properties which in turn modify the drying front length across the evaporating surface. Our result extends the understanding of physics of film failure which is relevant information for various industrial processes such as in inkjet printing and coating applications.
\end{abstract}




\section{Introduction}

The drying of colloidal dispersions is relevant to industrial applications such as the production of solid particulates, ceramics, catalysts, paints and pharmaceutical products as well as printing and coating processes. ${ }^{1-6}$ Typically consisting of solid particles dispersed in an aqueous solution, these materials evolve from a complex fluid into a brittle solid as they dry. The capillary forces raised by menisci between the colloidal particles are responsible for a build-up of internal stress during drying. This stress can be dissipated in several ways, most notably through the formation of various mechanical instabilities including cracks, ${ }^{7-9}$ shear bands ${ }^{10-12}$ and film peeling or delamination, ${ }^{13-15}$ which are all typically seen as commercially undesirable. Consequently, many studies have focused on the physics associated with the evaporation of colloidal dispersions, in order to prevent or control such mechanical instabilities. ${ }^{10-12,16-25}$ In particular, the occurrence of drying-induced cracks in thin films have been widely studied and a variety of methods for their prevention have been suggested to date. ${ }^{16,18,21-25}$ As a few examples: Singh et al. ${ }^{21}$ showed that by varying the flocculation state of a dispersion, through changes in the $\mathrm{pH}$ of the solution, the extent of cracking could be reduced; Jin et al. ${ }^{23}$ eliminated cracks by adding emulsion droplets to modulate the suspension viscosity; Similarly, Kappert et al. ${ }^{25}$ investigated the effects of organic colloids on cracking, which was reported to enhance the fracture resistance of coatings.

Although much literature has dealt with eliminating cracking in thin colloidal films, very few studies exist with regards to the prevention of drying-induced peeling of such films. ${ }^{26-28}$ Peeling occurs when the film detaches from its substrate, and a peeling film typically also curves upwards as it dries (as is commonly seen for example in drying clay or mud ${ }^{44}$ ). A Griffiths-like criterion for peeling can be made for when the strain energy stored in the film overcomes the adhesion energy holding the film in contact with the substrate. ${ }^{29}$ As an example of the 
complexity of this behaviour, Boulogne et al. ${ }^{26}$ investigated the effect of particle size and coating thickness on the drying kinetics of colloidal dispersions, and the resulting peeling of drying films. They reported a decrease in the early-stage curvature of peeling films with an increase in either parameter.

In general, the drying kinetics of colloidal dispersion proceeds in several stages. ${ }^{14,18,16,30,31,}$ In the initial phase, after a layer of the nanoparticle suspension has been spread or deposited onto a substrate, liquid evaporation occurs primarily from the surface, which concentrates the dispersion. As the bulk of the liquid continues to evaporate, a close packed film of particles begins to form, and the system starts to change from a fluid into a solid. At this point, for both drying droplets and more extended films, multiple different fronts will often appear from the edges of the deposit, and propagate inwards into a shrinking liquid pool (see Fig. 1). The tension of liquid menisci between primary particles in the wet (fluid-saturated) solid region of the drying film results in capillary stresses which are responsible for driving film delamination, amongst other mechanical instabilities. ${ }^{14,16,28,32}$ Thus, this region in particular, including for example its width and thickness, is central to understanding and controlling film peeling.

Here, motivated by how the evaporation of suspensions impacts diverse industrial applications, we investigate how film failure through peeling is influenced by the properties of a drying colloidal dispersion. Specifically, we will show that the drying-induced peeling of films can be reduced, or in fact eliminated, through changes in the viscosity of the dispersion, which in turn modify the extent of the active drying fronts. Our results extend the general understanding of film failure and are relevant to industrial processes such as drying paints, inkjet printing and coatings. 


\section{Theoretical considerations}

We consider now how a droplet or film of an aqueous colloidal dispersion will dry. As the liquid phase of the dispersion evaporates, the wet particles will come into contact to form a closely packed bed. Solidification of the colloidal dispersion typically begins at the vicinity of the droplet edge and proceed inwards towards the centre. ${ }^{33}$ The wet (fluid-saturated) solid region experiences stress, due to the capillary pressure exerted by the solvent-air menisci at the interface $^{18,34}$ which can lead to debonding of that region from the substrate. The initial properties of the colloidal dispersion can influence the drying dynamics and subsequent morphological instabilities, and we will show that the length of the wet solid region, where stresses develop, depends on the viscosity of the evaporating fluid. As a result, additives which enhance the dispersant's viscosity will modify the region of stress experienced across the solid deposit's surface, which in turn will influence the degree of film peeling.
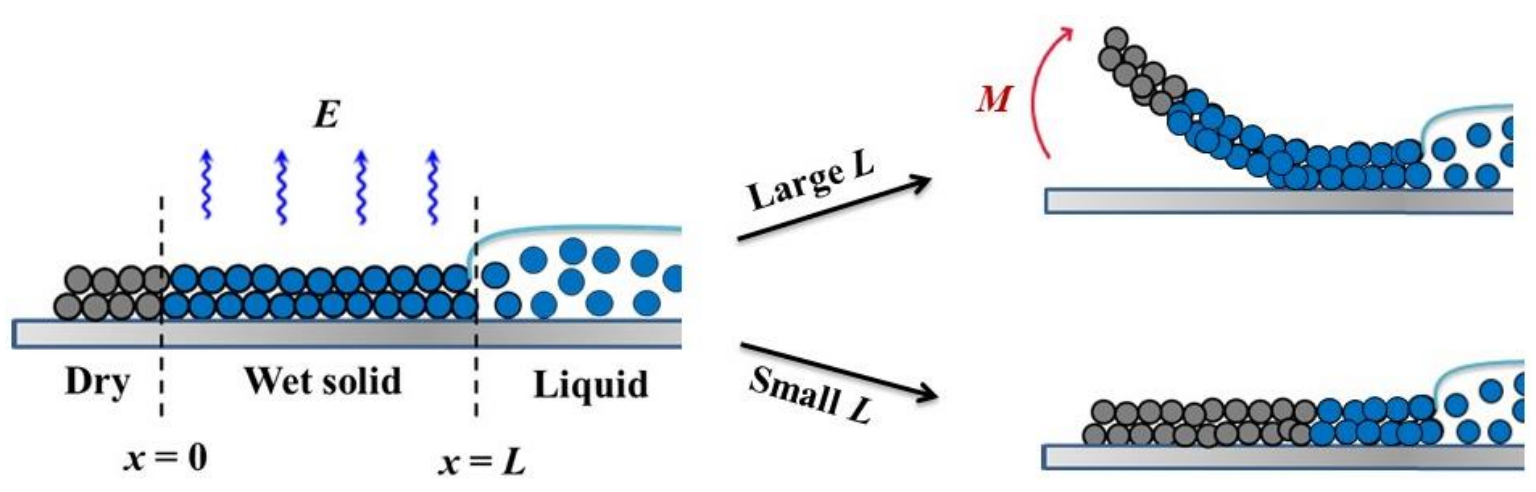

Figure 1. Conceptual sketch of the drying of a colloidal dispersion on a solid substrate. As the liquid evaporates from the surface the system undergoes a fluid to solid transition, which happens over a set of inward-moving drying fronts. We focus on two such fronts here: an inner front where the film solidifies into a rigid, but still wet film, and an outer front where the film desaturates and dries out. Between these fronts the colloidal films may delaminate from the substrate due to the built-up of stress, and a bending moment $M$. Here, the length of the wet solid region, $L$, is considered as a contributing factor in determining how the film debonds and 
bends. Widely spaced drying fronts (large $L$ ) encourage an out-of-plane displacement of the film, whilst a reduction in $L$ can eliminate such instabilities.

To understand how film peeling will depend on the viscosity of the dispersion, we assume that the drying geometry can be approximated by a pseudo-1D model and that the drying fronts (i.e. those defining the liquid-solid and desaturation/air-invasion transitions) are moving slowly compared to the flows of fluid within the wet porous medium. ${ }^{32,35}$ The relevant geometry and mechanisms governing the dynamics and the homogeneity of the final film are sketched in Figure 1. The droplet dries directionally such that it has an inner colloidal liquid that dries into a solid film, from the outside-in. The solid-liquid transition is relatively abrupt, and leaves a wet solid film in its wake. Additionally, there is another transition from this fluid-saturated solid to a dry solid, which lags behind the first front by some distance, $L$.

Between these fronts we consider a cross-section of the wet solid portion of the film, of length $L$ and height $h$; the air-invasion front is positioned at $x=0$ and the solid-liquid transition at $x=L$. Assuming that the film is fed by an inflow of water at speed $U_{0}$ from the droplet, and that there is a constant evaporation rate $E$ over the surface of the film, mass conservation implies that $U_{0}=E L / h$. As no fluid passes through either the final drying front or the substrate (at $z=0$ ) then, for an incompressible fluid, the superficial fluid velocity $\boldsymbol{U}$ throughout the film has components

$$
\left(U_{x}, U_{z}\right)=\left(-\frac{E x}{h}, \frac{E z}{h}\right)
$$

This flow through the pore space of the solidified colloidal film causes drag as it passes around the stationary particles, and the resulting change in the capillary pressure of the fluid, $P$, is given by Darcy's Law, 


$$
\nabla P=-\frac{\mu}{\kappa} \boldsymbol{U}
$$

where $\mu$ is the viscosity of the dispersant and $\kappa$ is the permeability of the solidified particle network. Considering first the $x$-component of the flows, Eq. (2) implies that

$$
\frac{\partial p}{\partial x}=-\frac{\mu}{\kappa} U_{x}=\frac{\mu E x}{\kappa h}
$$

which is solved by the pressure

$$
P=\frac{\mu E}{2 \kappa h}\left(x^{2}-L^{2}\right)+P(z)
$$

Here, we have set the pressure to be zero at the top point of the liquid-solid transition, i.e. at $x=L$ and $z=h$. The capillary pressure will reach an extreme value of $P_{\mathrm{c}}$ at the drying front, depending on how highly curved a meniscus can be while still fitting into the pore spaces of the drying film; it is generally accepted that $P_{\mathrm{c}}=-a \gamma / r$, where $a$ is a geometric constant of magnitude $\sim 5-10, \gamma$ is the surface tension of the fluid and $r$ is the radius of colloidal particles. ${ }^{18,34,36,37}$ At the top of the final drying front the pressure thus takes this well-defined value, and the width of the wet solid region of the film is predicted to be

$$
L=\sqrt{\frac{2 \kappa h P_{c}}{\mu E}} \sim \mu^{-1 / 2} .
$$

In section 4 we will present experiments that confirm this scaling of the wet film width, $L$, with the dispersant viscosity, $\mu$.

The forces discussed so far are frequently implicated in film fracture, including film debonding. ${ }^{18,33,34,38-41}$ However, they will not directly lead to the additional curling of a debonded film. Instead, this requires a bending moment, caused by a vertical distribution of forces, or pressures. The upwards flow caused by drying leads to such a pressure imbalance across the thickness of the film, given by 


$$
P(z)=\frac{\mu E}{2 \kappa h}\left(h^{2}-z^{2}\right)
$$

This pressure leads to the bending moment, $M$, as has been detailed elsewhere for drying films ${ }^{14,41-44}$. Briefly, this moment is the result of compressive or tensile stresses acting over the thickness of the wet solid film,

$$
M=\int_{0}^{h} \sigma_{x x}\left(z-\frac{h}{2}\right) d z
$$

where $\sigma_{x x}$ is the in-plane stress, and $(z-h / 2)$ is the distance away from the mid-line (i.e. neutral curve) at which this stress is applied. Assuming a linear poroelastic material, then the internal stress of the film is a response to the fluid pressure, and for a traction-free drying surface and an initially unstrained and unbent film $\sigma_{x x}=-P(z)(1-2 v) /(1-v)$, where $v$ is the film's Poisson ratio ${ }^{14,41-44}$. Under these conditions,

$$
\begin{gathered}
M=\int_{0}^{h} P(z)\left(\frac{1-2 v}{1-v}\right)\left(z-\frac{h}{2}\right) d z \\
=\frac{\mu E h^{3}}{24 \kappa}\left(\frac{1-2 v}{1-v}\right) .
\end{gathered}
$$

This moment will act to lift the film as shown in Figure 1, allowing the wet drying region to act as a hinge. ${ }^{15,41-43}$ It will also contribute to the stress intensity factor of an interfacial crack $^{44,45}$ although only weakly, as the pressures are a factor of $(h / L)^{2}$ smaller than those arising from the lateral flows (compare Eqs. $4 \& 6$ ). While the strength of the bending moment should increase with viscosity, it will also be expressed over a shorter distance, as $L \sim 1 / \sqrt{\mu}$ (Eq. 5). As we will now show, empirically, it is this shorter drying length which is more important in determining how the film debonds and bends. 


\section{Experimental methods}

Droplet drying experiments were performed using charge-stabilised dispersions of colloidal silica (Ludox HS, Grace) containing roughly spherical particles with a mean radius of $8 \mathrm{~nm} .{ }^{46}$ Various colloid-polymer dispersions were prepared by mixing polyethylene oxide (PEO, Sigma-Aldrich), polyvinylpyrrolidone (PVP, Sigma-Aldrich) or carboxymethyl cellulose (CMC, Sigma-Aldrich), of varying concentrations ( $0-2 \mathrm{wt} \%)$ and average molecular weight (0.04, 0.1, 0.6 and 1.0 MDa), with a $4 \mathrm{wt} \%$ silica dispersion (diluted using deionised water). Viscosity measurements of the resulting dispersions, containing varying polymer concentrations, were carried out by means of a stress-controlled rheometer (ARG2, TA Instruments). The measurements were repeated 20 times, and had a standard deviation of less than $1 \%$. Note that, to optimise the precision of the viscosity measurements, we measured the rheology of the dispersion, rather than the as-prepared dispersant (which was diluted in the process). Given the low initial particle concentration, this will lead to a slight $(<5 \%$ by the Einstein or Batchelor equation $)^{47}$ but consistent overestimation of the dispersant viscosity.

All droplet experiments were performed on cleaned microscope glass slides in ambient conditions (temperature $\sim 23^{\circ} \mathrm{C}$, relative humidity $\sim 36 \%$ ). Prior to use, the glass slides were cleaned for two hours in a strong surfactant solution (20\% by weight of Hellmanex III, Ossila, diluted with deionised water), rinsed, further cleaned with a solution of $1 \mathrm{M} \mathrm{NaOH}$, and rinsed again thoroughly with deionised water. ${ }^{48} \mathrm{~A}$ constant evaporation rate was confirmed by measuring droplet mass loss over time of pure water and droplets of colloid-polymer suspensions. In all cases, no changes in the evaporation rate was observed.

To visualise the drying dynamics, $3 \mu \mathrm{L}$ drops of dispersion were placed on slides using a micropipette and top-view images were taken with an optical microscope (Leica M205C) 
throughout the evaporation process. In addition, an automatic imaging system was set up to record a side-view image sequence of each evaporating drop using a digital camera (Canon EOS 700D). The resulting images were manually processed using Image ${ }^{49}$ to obtain the radius of the curvature of the peeled films. The curvature, defined as $1 / r$ where $r$ is the radius of curvature of the film was computed by fitting a circle around the outside of the curved film. For consistency, the final side-view images captured towards the end of the drying process (just before the central liquid region finally disappeared) were analysed. Each experimental condition was repeated 10 times to ensure the reproducibility of the data, and average values of the curvature are reported in the results of this study.

\section{Results and discussion}

\subsection{Evaporation of colloidal droplets}

A series of images qualitatively illustrating how a representative colloidal droplet evolves as drying proceeds is shown in Figure 2. Initially, the drying resembles that of a pure droplet, i.e. a constant decrease in volume as the bulk of the liquid evaporates. ${ }^{14}$ However, as the drying process continues, solidification of the suspension begins at the vicinity of the droplet edge and pins the film in place (Fig. 2(a)). Multiple different fronts then advance into the interior of the droplet, which can be identified through changes in transparency or the appearance of a parallel array of cracks, ${ }^{25}$ as seen in Fig. 2(b,c). The innermost such front shows where the film solidifies into a rigid, but still wet film, while the outermost front indicates where the film desaturates, and dries out. Between these fronts the colloidal films may delaminate from the substrate due to the built-up of stress. Once debonded, further evaporation-driven stresses cause the film to bend and curl upwards, as seen towards the end of drying process (Fig. 2(d)). 

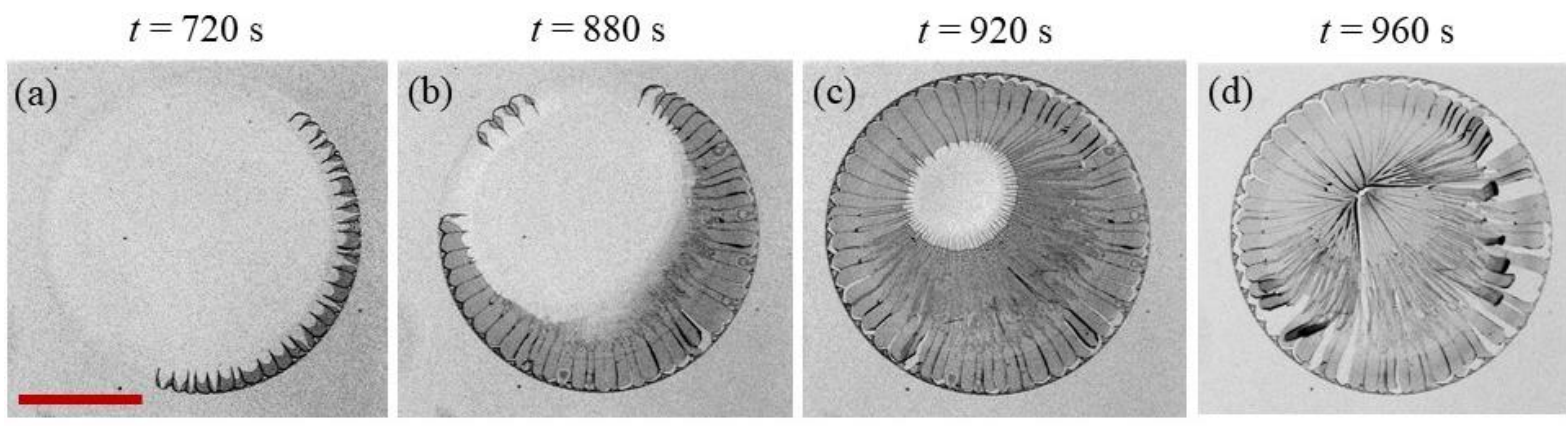

Figure 2. Typical images captured at different times and illustrating the evolution and solidification of a nanofluid droplet containing 4\% silica particles and $0.01 \%$ PEO (M.W. of $0.1 \mathrm{MDa})$. The initial droplet volume was $3 \mu \mathrm{L}$. The time $t$ indicates the elapsed time since deposition and the scale bar represents $1 \mathrm{~mm}$.

In order to study the effect of the viscosity of the fluid on the drying dynamics and film peeling, PEO of varying concentration and molecular weight was added to the dispersion (see Experimental Methods). Colloid-polymer droplets were then deposited on glass substrates and left to dry. Figure 3 shows the final film characteristics obtained with varying concentration and molecular weight. It is clear from the captured images that an increase in the fluid viscosity, due to the presence of the polymer additive, does influence the extent of the film peeling instability. 


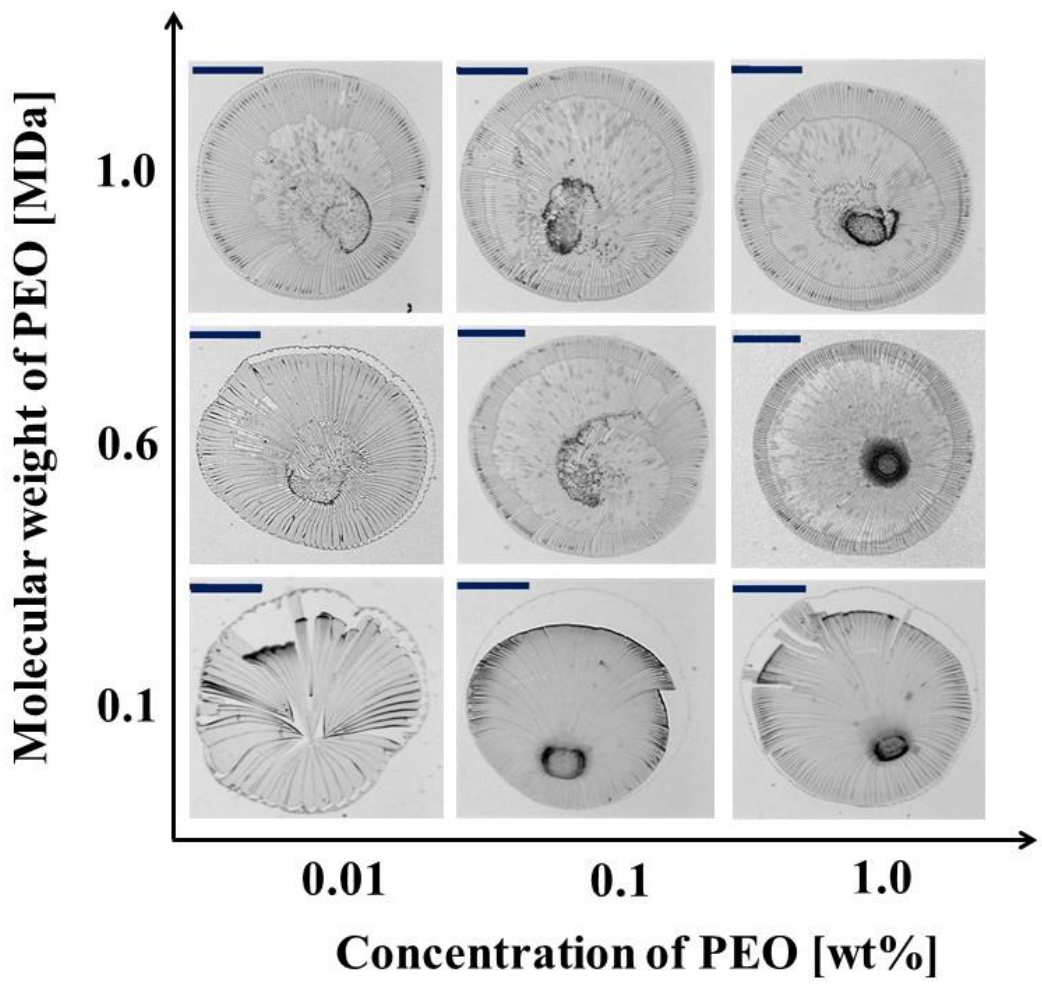

Figure 3. The nature of the final dried films obtained from the drying of colloid-polymer dispersion depends on the molecular weight and concentration of the polymer: additional polymer, or a longer chain-length, tends to discourage film peeling. The scale bars represent 1 $\mathrm{mm}$.

In the case of droplets containing a low PEO concentration of low molecular weight, the drying behaviour is similar to that of pure silica dispersions i.e. droplet evaporation leads to significant film peeling (Fig. 2). However, as the PEO concentration and molecular weight increases the extent of peeling is reduced, and it is eliminated with a large enough increase in these parameters (Fig. 3). We argue that the notable decrease in the peeling instability of colloidal films with the addition of PEO is due to the changes in the viscosity of the dispersant fluid, which increases with the polymer concentration and molecular weight. To this end, Fig. 4 shows the viscosity measurements of the dispersions plotted against the measured curvature of the corresponding films just before the final dried state. Additional droplet drying experiments were conducted using different additives, and their data are also included in Fig. 4. The 
resulting data collapse, regardless of whether experiments were done with polymers of different composition, molecular weight or concentration demonstrates that the elimination of peeling is a universal effect, regardless of the exact nature of the thickening agent used.

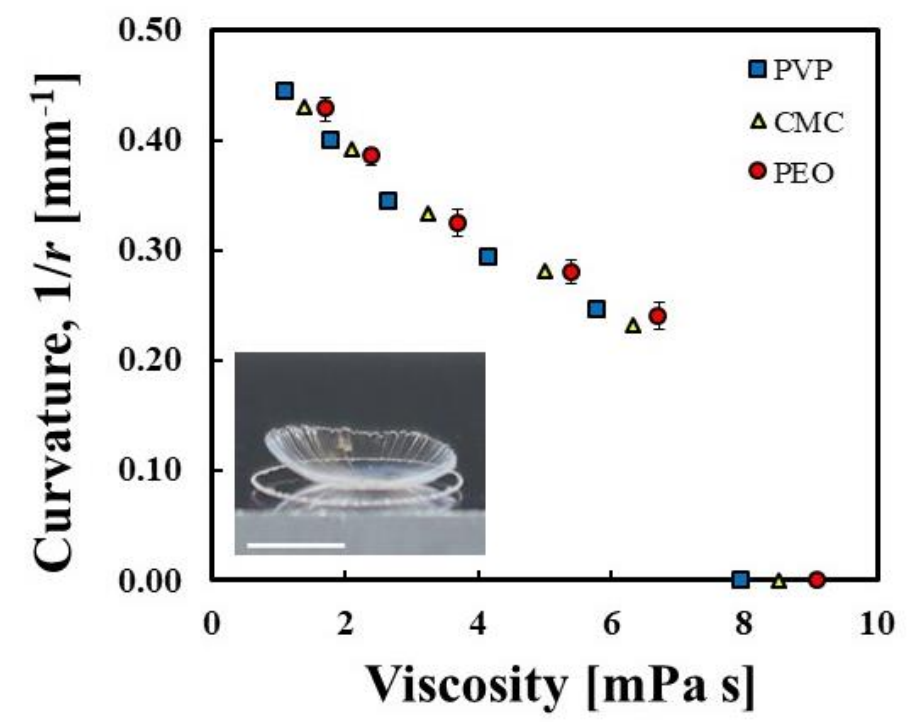

Figure 4. Viscosity measurements of the colloid-polymer dispersions plotted against the obtained curvature, $1 / r$, of the corresponding delaminated films. A gradual increase in the viscosity tends to discourage peeling and eventually leads to its complete arrest. The inset image shows typical example of the peeled films and the scale bar represents $2 \mathrm{~mm}$.

The strong correlation seen in Figure 4 confirms that the extent of peeling is indeed influenced by the viscosity of the dispersion, such that a gradual increase in the viscosity eventually leads to its complete arrest. To validate the above mechanism (Figure 1) and the relationship presented in eq. (5), i.e. that the drying front width is inversely proportional to the fluid viscosity, we experimentally visualised and measured the length of the drying front in all cases. To do so, the droplet dispersions were initially left to partially dry (Figure 5a) after which a small amount blue water dye (Oasis) was added to the centre of the evaporating droplets as shown in Figure 5b. In general, this dye will then sweep through the wet rigid film, highlighting the region between the various drying fronts, ${ }^{50}$ which allowed the visualisation of the drying 
front, $L$, (indicated by the dashed line in Figure 5c). This region was measured to obtain the equivalent diameter through image analysis using ImageJ at the end of the drying process.

$t=910 \mathrm{~s}$

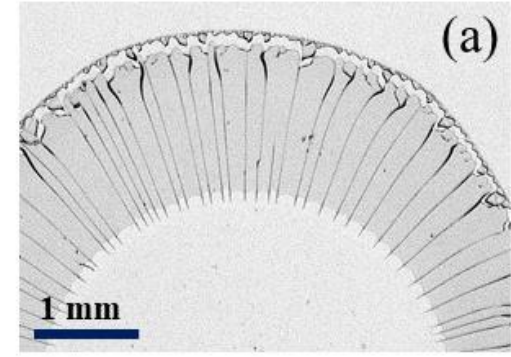

$t=1000 \mathrm{~s}$

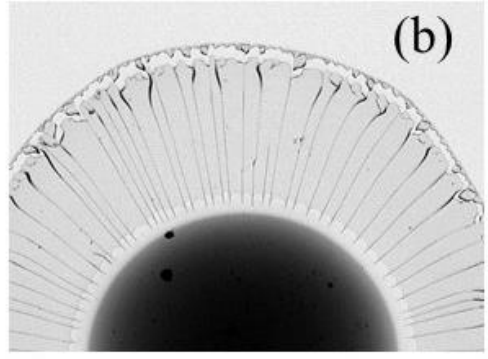

$$
t=1090 \mathrm{~s}
$$

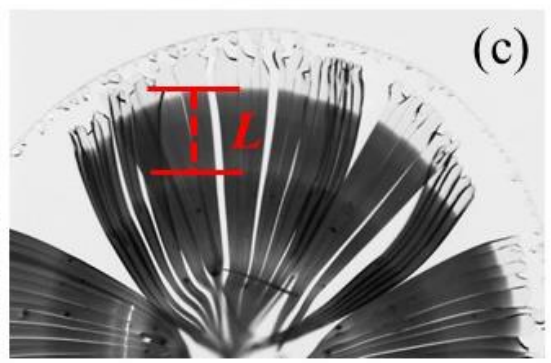

Figure 5. Images of a partially dried colloidal droplet (a) the partially dried colloidal droplets + water dye (b) the final dried films + water dye (c). Dye was used to highlight the region between the various drying fronts. At the end of the experiment, the surface of the films is stained by the dye as seen in Figure $5 c$. The central region usually remains the darkest compared to other regions since this was the initial point of dye deposition.

Figure 6 shows the drying front width, $L$, compared with its expected dependence on the fluid viscosity. We can measure the slope of this relationship, $c$, from our experimental data (Figure 6) to be $\sim 6 \times 10^{-5} \mathrm{~m}(\mathrm{~Pa} \mathrm{~s})^{-1 / 2}$. This agrees well with the value of $c=8 \times 10^{-5} \mathrm{~m}(\mathrm{~Pa} \mathrm{~s})^{-1 / 2}$ estimated using Eq. (5), assuming $P_{c}=5.3 \gamma / R,{ }^{18,34,37}$ for typical values of $\kappa \approx 5 \times 10^{-20} \mathrm{~m}^{2}$ (calculated using the Carman-Kozeny equation $\kappa=R^{2}(1-\phi)^{3} / 45 \phi^{2}$ and a final volume fraction of 0.74$), \gamma=0.07$ Pa.m, $R=8 \times 10^{-9} \mathrm{~m}, h=3 \times 10^{-5} \mathrm{~m}$ and $E \approx 2 \times 10^{-8} \mathrm{~m} / \mathrm{s}$. Additionally, the coefficient of determination $\left(\mathrm{R}^{2}\right)$ for the linear fit of the experimental values is 0.97 , which corroborates the proposed mechanism presented in Figure 1 and Eq. (5), that is, the length of the drying front is inversely proportional to the square root of the viscosity of the evaporating fluid, which consequently affects the extent of film peeling from solid substrates. 


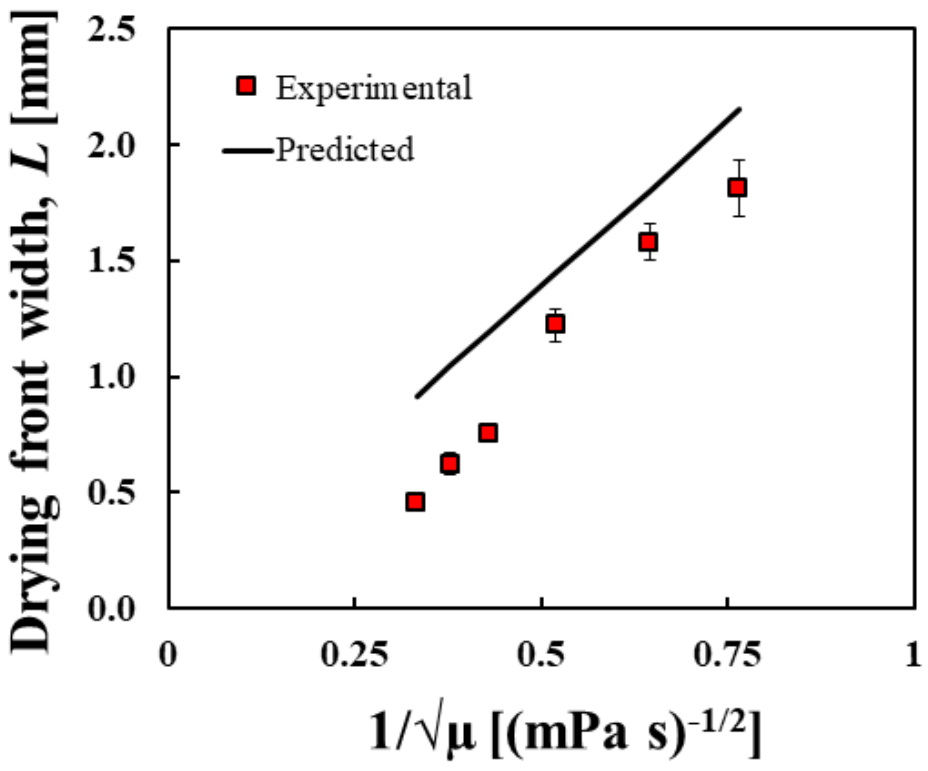

Figure 6. The measured length of the drying front, $L$, is related to the dispersion viscosity, $\boldsymbol{\mu}$. In all cases, additional polymer, or a longer chain-length, tends to discourage film peeling. The predicted values are calculated using Eq. 5 and material parameters as described in the text.

\section{Conclusion}

The aim of the present work was to investigate the drying dynamics of colloidal dispersions and the resulting characteristics of the film formed at the end of drying, specifically its ability to remain well-bound to its substrate. To do so, we conducted a comprehensive series of droplet experiments with aqueous mixtures of silica nano-dispersions and polymers additives. Timelapse digital microscope images of the droplets show how an increase in the molecular weight and concentration of the polymer reduces the curvature of the peeled films and eventually leads to its complete arrest. This is the result of the additives changing the dispersion properties, in particular the viscosity of the evaporating solution, which in turn modifies the length of the drying front, and the distribution of forces active during drying. The presence of such polymer will not have a significant effect on surface adhesion due to the dilute dispersion used in this 
work. It is likely that the polymer interaction with the surface is limited, in such case. To our knowledge, little work has been done in the literature on this topic and thus, a greater focus of the adhesive and cohesive interactions experienced by thin film in the presence of such additives would be interesting. The findings of this research will be useful for controlling the drying induced peeling of films by simply modifying the initial properties, which could be useful in a variety of applications including inkjet printing, ceramics and coating technologies.

\section{Acknowledgements}

We would like to acknowledge the UK Engineering and Physical Sciences Research Council (EPSRC) to provide the PhD studentship for Abdulkadir Osman and Johnson Matthey for additional funding of this project. All experiments reported in this paper were conducted at The University of Manchester.

\section{References}

(1) Osman, A.; Shahidzadeh, N.; Stitt, H.; Shokri, N. Morphological transformations during drying of surfactant-nanofluid droplets. J. Ind. Eng. Chem. 2018, 67, 92-98.

(2) Keck, S. Mechanical alteration of the paint film. Stud. Conserv. 1969, 14, 9-30.

(3) Rahaman, M. N. Ceramic Processing and Sintering; Marcel Dekker Inc: New York, 1995.

(4) Parisse, F.; Allain C.; Drying of Colloidal Suspension Droplets: Experimental Study and Profile Renormalization. Langmuir 1997, 13, 3598.

(5) Vehring, R. Pharmaceutical particle engineering via spray drying. Pharm. Res. 2008, 25, 999-1022.

(6) Deegan, R. D., Bakajin, O., Dupont, T. F., Huber,G., Nagel. S. R. \& Witten, T. A. Capillary flow as the cause of ring stains from dried liquid drops. Nature 1997, 389, 827-829 
(7) Shokri, N.; Zhou, P.; Keshmiri, A. Patterns of Desiccation Cracks in Saline Bentonite Layers. Transp. Porous Media 2015, 110, 333-344.

(8) Goehring, L.; Clegg, W. J.; Routh, A. F. Wavy cracks in drying colloidal films. Soft Matter 2011, 7, 7984 .

(9) Dufresne, R.; Stark, D. J.; Greenblatt, N. A.; Cheng, J. X.; Hutchinson, J. W.; Mahadevan, L.; Weitz, D. A. Dynamics of Fracture in Drying Suspensions. Langmuir 2006, 22, 7144.

(10) Kiatkirakajorn, P.-C.; Goehring, L. Formation of Shear Bands in Drying Colloidal Dispersions. Phys. Rev. Lett. 2015, 115, 088302.

(11) Yang, B.; Sharp, J.S.; Smith, M.I. Shear banding in drying of films of colloidal nanoparticles. ACS Nano, 2015, 9, 4077-4084

(12) Yang, B.; Smith, ND,; Johannes, A.; Burghammer, M.; Smith, MI. Shear bands and the evolving microstructure in a drying colloidal film studied with scanning $\mu$-SAXS. Sci. Rep. 2018, $8(1), 12979$.

(13) Lazarus, V.; Pauchard, L. From craquelures to spiral crack patterns: influence of layer thickness on the crack patterns induced by desiccation. Soft Matter 2011, 7, 2552.

(14) Giorgiutti-Dauphinéa, F.; Pauchard, L. Colloids Surf. A Physicochem. Eng. Asp. Dynamic delamination of drying colloidal films: Warping and creep behaviour. 2015, 466, 203-20.

(15) Sefiane, K. Patterns from drying drops. Adv. colloid interfac. 2014, 206, 372-381.

(16) Giorgiutti-Dauphinéa, F.; Pauchard, L. Drying drops. Eur. Phys. J. E 2018 41: 32

(17) Osman, A.; Goehring, L.; Patti, A.; Stitt, H.; Shokri. N. Fundamental investigation of the drying of solid suspensions, Ind. Eng. Chem. Res. 2017, 56, 10506-10513. 
(18) Routh, A. Drying of thin colloidal films. 2013, Rep. Prog. Phys. 76, 046603

(19) Osman, A.; Leaper, S.; Sreepal, V.; Gorgojo, P.; Stitt, H.; Shokri, N. Dynamics of Salt Precipitation on Graphene Oxide Membranes. Cryst. Growth. Des. 2019, 19, 498-505.

(20) Deegan, R. D., Bakajin, O., Dupont, T. F., Huber,G., Nagel. S. R. \& Witten, T. A. Contact line deposits in an evaporating drop. Phys. Rev. E 2000, 62, 756-762.

(21) Singh, K. B.; Bhosale, L. R.; Tirumkudulu, M. S. Cracking in drying colloidal films of flocculated dispersions. Langmuir 2009, 25, 4284-4287.

(22) Hagan, E. W. S.; Charalambides, M. N.; Young, C. R. T.; Learner, T. J. S.; Hackney, S. Viscoelastic properties of latex paint films in tension: Influence of the inorganic phase and surfactants. Prog. Org. Coat. 2010, 69, 73-81.

(23) Jin, Q.; Tan, P.; Schofield, A. B; Xu, L. Eliminating cracking during drying. Eur. Phys. J. E 2013, 36, 28.

(24) Wang, L.; Zhao, X. S. Fabrication of crack-free colloidal crystals using a modified vertical deposition method. J. Phys. Chem. C 2007, 111, 8538-8542.

(25) Kappert, E. J.; Pavlenko, D.; Malzbender, J.; Nijmeijer, A.; Benes, NE.; Tsai, PA. Formation and prevention of fractures in sol-gel-derived thin films. Soft Matter 2015, 11, 882888.

(26) Boulogne, F.; Stone. H.A. Self-crumpling elastomers: bending induced by the drying stimulus of a nanoparticle suspension. Europhys Lett 2014, 108, 19001.

(27) Yang, Bin.; Sharp, J.S.; Smith, M.I. The interplay of crack hopping, delamination and interface failure in drying nanoparticle films. Sci Rep. 2016, 6, 32296. 
(28) Sarkar, A.; Tirumkudulu, M.S. Delamination of drying nanoparticle suspensions. Soft Matter, 2011, 7, 8816.

(29) Pauchard, L. Patterns caused by buckle-driven delamination in desiccated colloidal gels. Europhys Lett 2006, 74, 188-194.

(30) Bacchin, P et al. Drying colloidal systems: Laboratory models for a wide range of applications. Eur. Phys. J. E 2018 41, 94

(31) J. L. Keddie and A. F. Routh, Fundamentals of latex film formation. Processes and Properties, Springer, 2010.

(32) Kralchevsky, P.A.; Nagayama, K.. Capillary forces between colloidal particles Langmuir 1994 10, 23-36.

(33) Goehring, L.; Clegg, WJ.; Routh, AF. Solidification and Ordering during Directional Drying of a Colloidal Dispersion Langmuir 2010 26, 9269-9275

(34) Scherer, GW. Crack-tip in gels. J. Non-Cryst. Solids 1992, 144, 210-216

(35) Routh, A. F. Russel, W. B. Horizontal drying fronts during solvent evaporation from latex films. AIChE 1998, 44, 9.

(36) White, L.R. Capillary rise in powder. J. Colloid Interface Sci. 1982, 90, 536-538.

(37) Dufresne, E. R.; Corwin, E. I.; Greenblatt, N. A.; Ashmore, J.; Wang, D. Y.; Dinsmore, A. D.; Cheng, J. X.; Xie, X. S.; Hutchinson, J. W.; Weitz D. A. Flow and fracture in drying nanoparticle suspension. Phys. Rev. Lett. 2003, 91, 224501

(38) Hutchinson, J.W.; Suo, Z. Mixed mode cracking in layered materials. Adv. Appl. Mech. 1991, 29, 63-191. 
(39) Chiu, R.C.; Cima, M.J. Drying of Granular Ceramic Films: II, Drying Stress and Saturation Uniformity. J. Am. Ceram. Soc. 1993, 76, 2769-2777.

(40) Man, W.; Russel WB. Direct measurements of critical stresses and cracking in thin films of colloid dispersions. Phys. Rev. Lett 2008, 100, 19830

(41) Goehring L, Nakahara A, Dutta T, Kitsunezaki S, Tarafdar S. 2015 Desiccation cracks and their patterns: formation and modelling in science and nature. Singapore: Wiley-VC

(42) Scherer, G.W. Drying gels: iii. Warping plate. J. Non-Cryst. Solids 1987, 91, 83-100.

(43) Scherer, G.W. Drying gels viii. Revision and review. J. Non-Cryst. Solids 1989, 109, 171182.

(44) Style, R. W.; Peppin, S. S. L. Cocks, A. C. F. Mud peeling and horizontal crack formation in drying clays. J. Geophys. Res. 2011, 116, F01025.

(45) Thouless, M. D.; Evans, A. G.; Ashby, M. F.; Hutchinson, J. W. The Edge Cracking and Spalling of Brittle Plates. Acta Metallurgica, 1987, 35, 1333-1341

(46) Goertz, V.; Dingenouts, N.; Nirschl, H. Comparison of Nanometric Particle Size Distributions as Determined by SAXS, TEM and Analytical Ultracentrifuge Part. Part. Syst. Char. 2009, 26, 17-24.

(47) Batchelor, G.K. J. Fluid Mech., Vol. 83, 1, pp. 97-117, 1977

(48) Hemmerle, A.; Schröter, M.; Goehring, L. A cohesive granular material with tunable elasticity. 2016, Sci. Rep. 6, 35650

(49) Schneider, C.A., Rasband, W.S., Eliceiri, K.W. "NIH Image to ImageJ: 25 years of image analysis". Nature Methods 9, 671-675, 2012. 
(50) Laval, C.; Bouchaudya, A.; Salmon, JB. Fabrication of microscale materials with programmable composition gradients. Lab Chip, 2016, 16, 1234-1242.

\section{Table of Contents Graphic}
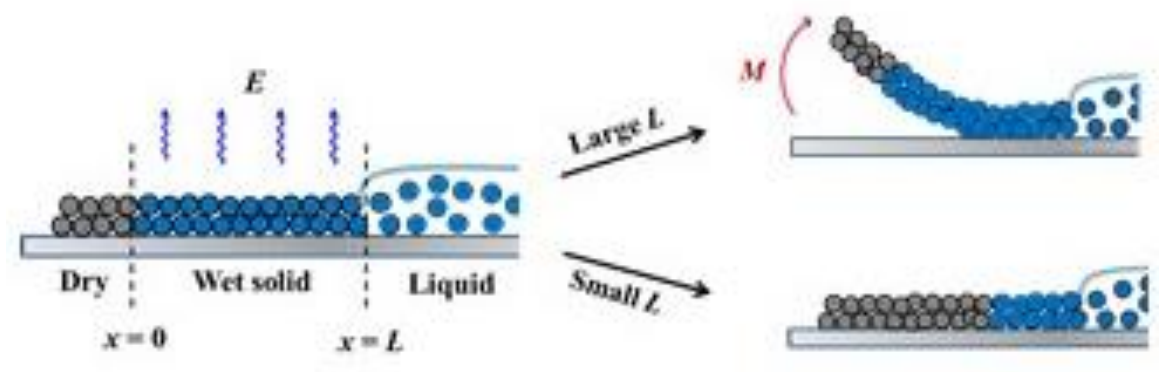

Controlling film peeling through modifying the properties of the evaporating solution and consequently the length of the drying front. 